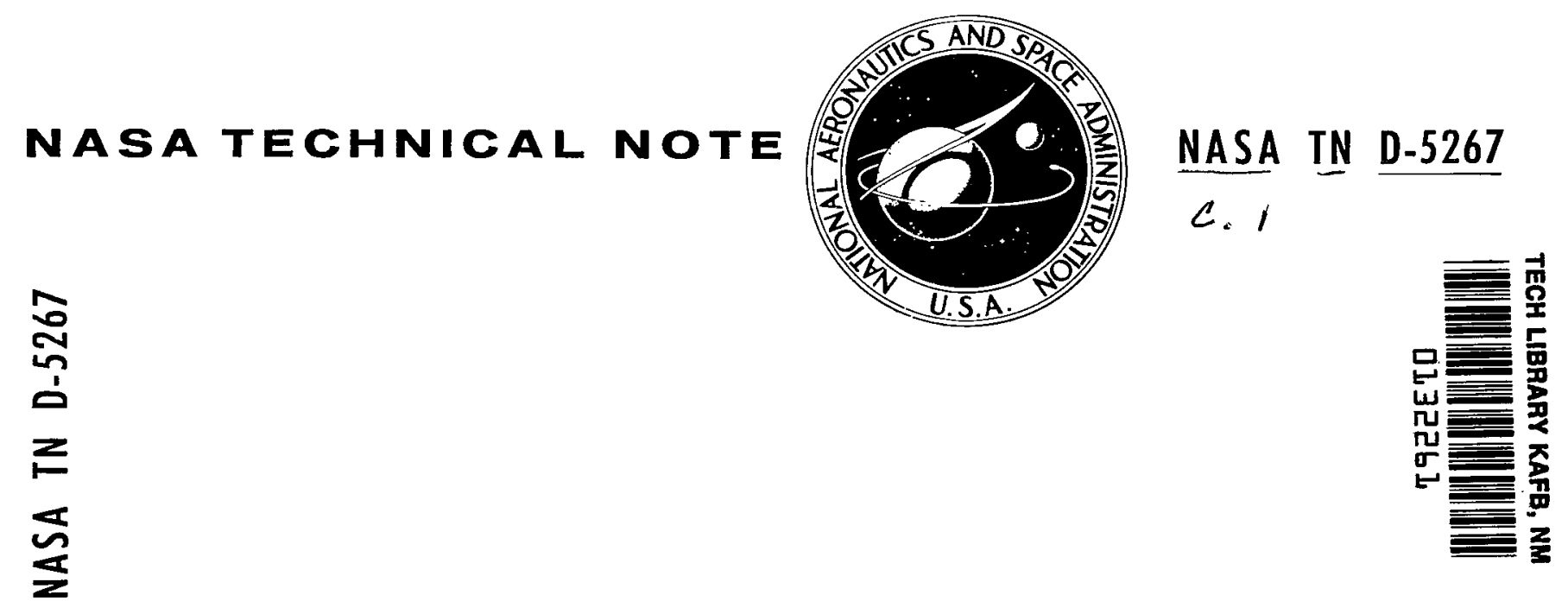

LOAN COPY: RETURN TO AFWL (WLIL-2) KIRTLAND AFB, N MEX

\title{
THE PIONEER 8 COSMIC DUST EXPERIMENT
}

by Otto E. Berg and F. Frank Richardson

Goddard Space Flight Center

Greenbelt, $M d$.

NATIONAL AERONAUTICS AND SPACE ADMINISTRATION • WASHINGTON, D. C. • JULY 1969 
THE PIONEER 8 COSMIC DUST EXPERIMENT

By Otto E. Berg and F. Frank Richardson

Goddard Space Flight Center

Greenbelt, Md.

\section{NATIONAL AERONAUTICS AND SPACE ADMINISTRATION}

For sole by the Cleoringhouse for Federal Scientific and Technical Informotion Springfield, Virginio 22151 - CFSTI price $\$ 3.00$ 


\begin{abstract}
A cosmic dust sensor comprising a unique array of sensors, sensor controls, and in-flight calibrations was placed in a heliocentric orbit (0.99 to 1.088 a.u.) on December 13, 1967, on board the Pioneer 8 satellite. The ionization and momentum imparted by the impact of a cosmic dust particle upon a surface are measured by the sensor to determine the particle's direction, speed, and mass. Prelaunch calibration studies using the Goddard Space Flight Center electrostatic accelerator have shown that the sensors can measure kinetic energies of impacting particles as low as $0.6 \mathrm{erg}$ and momenta as low as $2 \times 10^{-5}$ dyne-sec.
\end{abstract}




\section{CONTENTS}

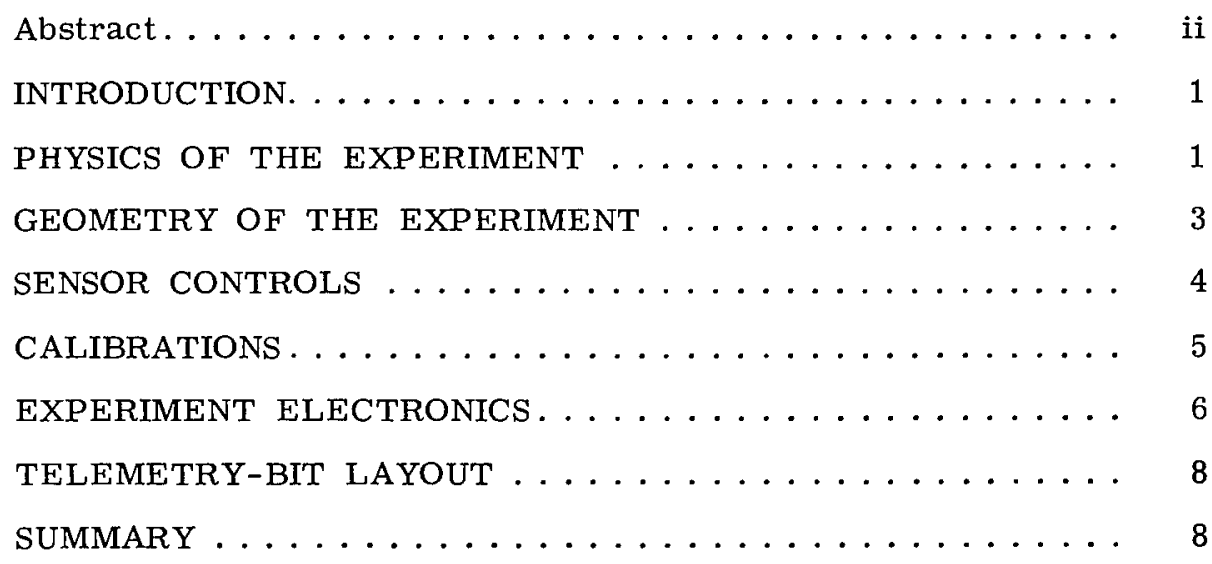




\title{
THE PIONEER 8 COSMIC DUST EXPERIMENT
}

\author{
by
}

\author{
Otto E. Berg and F. Frank Richardson
}

Goddard Space Flight Center

\section{INTRODUCTION}

One of the seven experiments on board the Pioneer 8 satellite is a cosmic dust experiment, beginning a new generation of cosmic dust sensors. It uses a unique array of individual detectors, detector controls, and electronic monitors designed to yield data that could justify a high degree of confidence and command a high degree of experimental integrity.

The objectives of the experiment are

1. To measure the cosmic-dust flux density in the solar system,

2. To determine the distribution of cosmic dust concentrations (if any) in the earth's orbit,

3. To determine the radiant flux density and speed of particles in meteor streams, and

4. To perform an in-flight control experiment on the reliability of the microphone as a cosmic dust sensor.

Pioneer 8 was launched on December 13, 1967, into a heliocentric orbit with perihelion and aphelion distances of 0.99 and 1.088 a.u., respectively and an anticipated operating lifetime of 1 year. The spin rate is approximately $1 \mathrm{rps}$ about an axis perpendicular to the ecliptic plane. The experiment is mounted in the equator of the satellite with its axis radial to the satellite spin axis.

\section{PHYSICS OF THE EXPERIMENT}

The experiment, shown schematically in Figure 1, consists of two film-grid sensor arrays spaced $5 \mathrm{~cm}$ apart (film plane to film plane) and an acoustical impact plate upon which the rear film is mounted. The performance of the sensors depends upon two measurable phenomena that occur when a hypervelocity particle impacts upon a surface-the formation of an ionized plasma and a transfer of momentum. 
In conjunction with the following explanation of the operation of the experiment, consider three probable cosmic dust particle types:

1. High-energy, hypervelocity particles (>1.0 erg),

2. Low-energy, hypervelocity particles $(<1.0 \mathrm{erg})$; and

3. Relatively large high-velocity particles ( $>10^{-10}$ grams).

As a high-energy, hypervelocity particle enters the front film sensor, it yields some of its kinetic energy toward the generation of ionized plasma at the front, or "A," film. The electrons from the plasma are collected on the positively

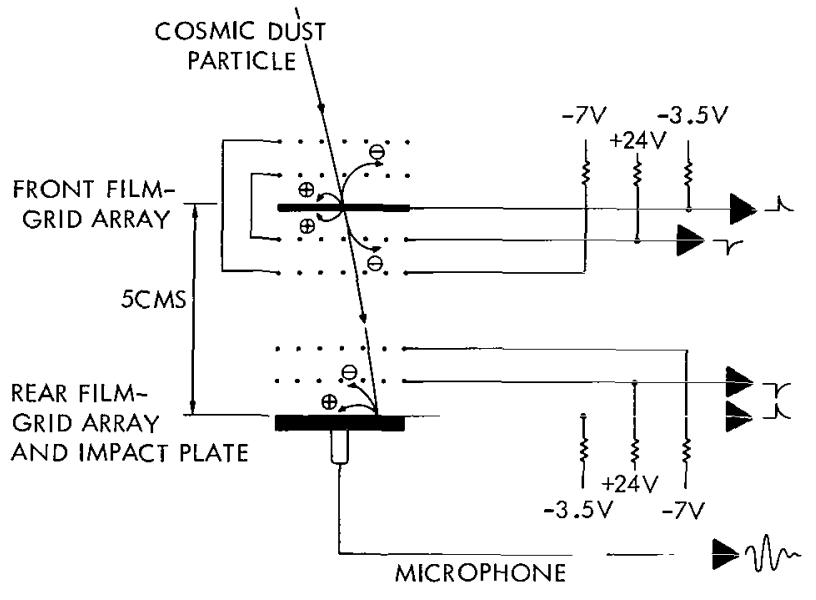

Figure 1-Schematic diagram of the sensor. biased grid ( +24 volts) initiating amplified negative pulses as shown. The ions from the plasma are collected on the negatively biased film ( -3.5 volts) producing a positive-going pulse that is amplified as shown and pulse-height-analyzed (PHA) as a measure of the particle's kinetic energy at the rear sensor, or "B," film and plate, generating a second set of plasma pulses and an acoustical pulse (i.e., if particle momentum is sufficient). A peak pulse height analysis (PPHA) is performed on the acoustical sensor output as a measure of the particle's remaining momentum.

As a low-energy, hypervelocity particle enters the front sensor, it yields all of its kinetic energy at the "A" film. A PHA is performed on the positive output signal to measure the particle's kinetic energy. A high-energy hypervelocity particle may be erroneously registered as a low-energy hypervelocity particle if, because of its angle of entry into the experiment, it fails to impact on the "B" film.

As a relatively large, high-velocity particle enters the experiment, it may pass through the front and rear film sensor arrays without generating a detectable ionized plasma; but it may still impart a measurable impulse to the acoustical sensor.

An electronic "clock" registers the time of flight (TOF) of the particle. The time lapse between positive pulses ("A" and "B" film output signals) is used to derive particle speed.

The TOF sensor is one of 256 similar sensors (including 31 control sensors) that comprise the portion of the Pioneer 8 experiment measuring particle speed and direction. Figure 2 is an exploded schematic view of the overall experiment. It shows the four vertical film strips crossed by four horizontal grid strips that effect 16 front and 16 rear film sensor arrays (each 2.5 by $2.5 \mathrm{~cm}$ ) and create the 256 total possible combinations. Each grid strip and film strip connects to a separate output amplifier. The output signals from these amplifiers are used to 

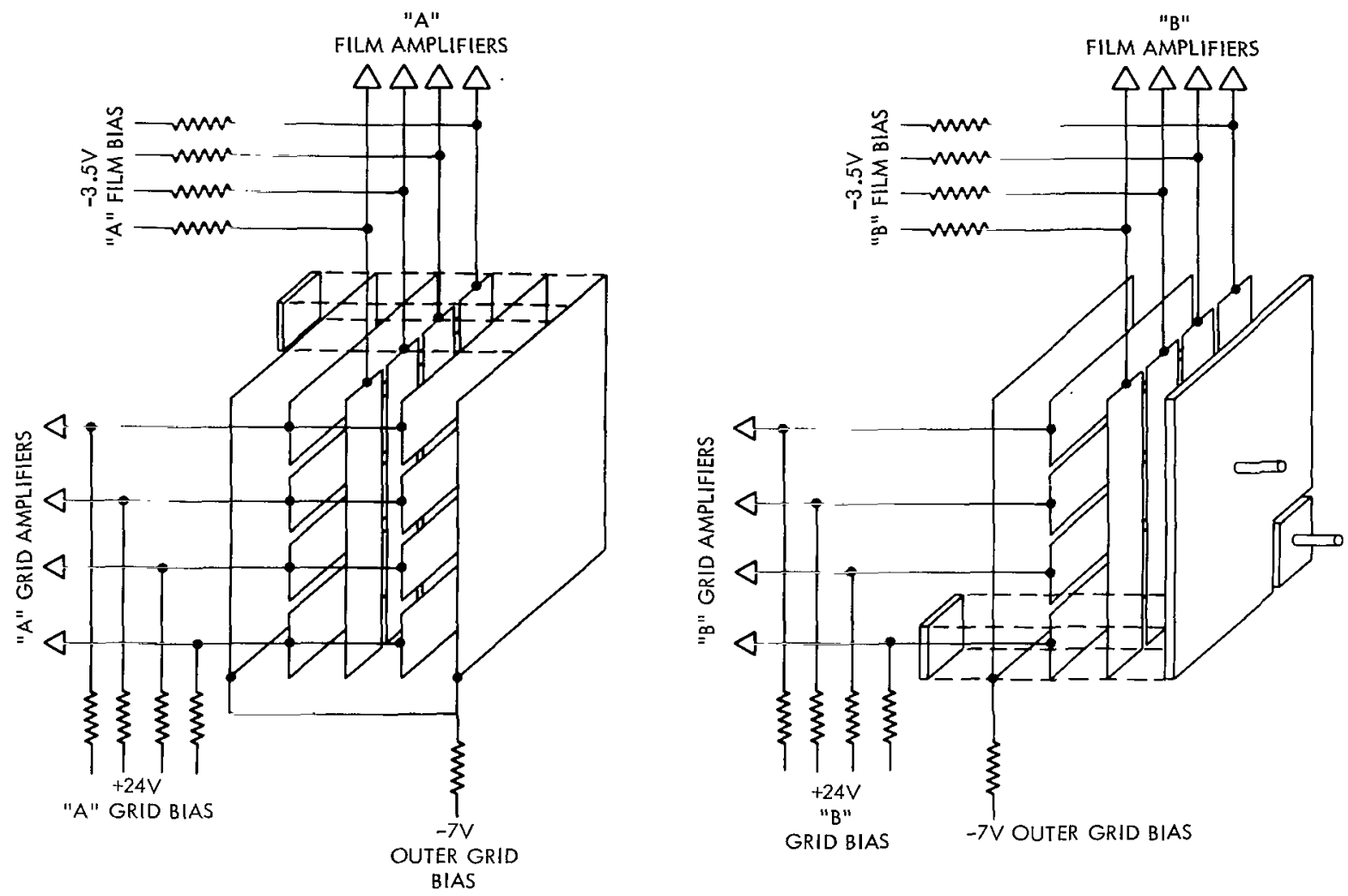

Figure 2-Schematic diagram of the sensor arrays.

determine the segment in which an impact occurred. Thus, by knowing the front film-grid segment penetrated and the rear film-grid segment affected by the impact, one can determine the direction of the incoming particle with respect to the sensor axis and the spacecraft attitude. A solar aspect sensor in the spacecraft is used to determine the sun-to-spacecraft angle at the time of an impact. This readout is initiated by an impact event involving the "A" film and/or the "B" film and/or the mic rophone.

\section{GEOMETRY OF THE EXPERIMENT}

Each of the four vertical films of the front sensor array, as shown in Figure 2, is a composite of eight layers as shown in an exploded view in Figure 3. Ideally, a thin copper foil (500 ̊) would be used alone for the vertical strips of the front sensor array, ${ }^{1}$ but the foil is obviously too fragile and subject to corrosion. Therefore, a nickel grid, the parylene ${ }^{2}$ substrate, and the parylene encapsulation are used as supports and anti-corrosion covering for the metal film deposits. The aluminum layers, serving only as fabrication aids during the preparation of the composite film, reflect the intense heat generated by copper evaporation upon the parylene substrate.

\footnotetext{
I Target materials, such as copper, molybdenum, and tantalum, have been found, by experiment, to enhance the formation of plasma from hypervelocity impacts.

2 A patented product of the Union Carbide Corporation.
} 


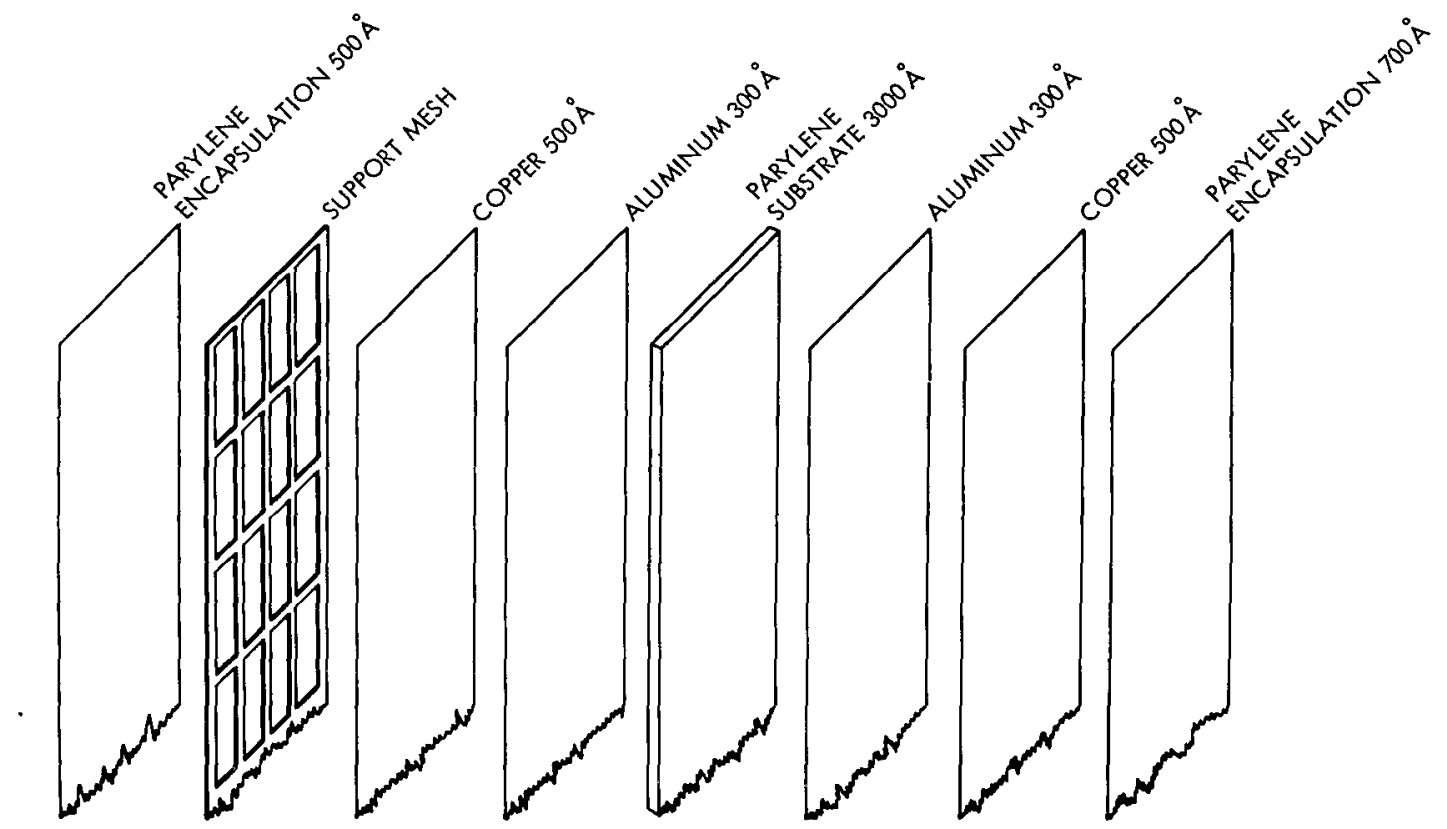

Figure 3-Suspended front-film configuration.

Each of the rear sensor array film strips is a $60 \mu$ molybdenum sheet cemented to a quartz acoustical sensor plate. The optical transparency of each of the grids (including support mesh) is 98.8 percent.

The maximum field of view of the experiment is that of the front film array and is square with a half angle of 60 degrees yielding a 4.5 steradian field. ${ }^{3}$ The minimum angular resolution of each TOF detector (see Figure 1 ) is \pm 27 degrees.

\section{SENSOR CONTROLS}

An ideal sensor control is one that is exposed to the same "environment" as the active or main sensor. "Environment" encompasses electrical and magnetic radiation, thermal radiation, and thermal gradients. Controls installed somewhere in the spacecraft, and sheltered from the total environment are ineffective. The controls used in this experiment were designed to perform under the same conditions as the main sensor as much as possible.

A simplified diagram of the overall experiment and the position of the sensor controls is shown in Figure 4. The upper. left segment of the front film-grid array and the upper right segment of the rear film-grid array are used as controls for the ionization sensors. The controls film and grid are potted in an epoxy resin that isolates them from the products of ionization caused by impacts upon their areas (i.e., electrons and ions generated by hypervelocity impacts upon the epoxy cannot be collected on the grids or films). The resin coat does not, however, constitute a

$\sqrt{3}$ The front film is recessed $3 \mathrm{~cm}$ into the experiment housing. 
shield from electrical or magnetic radiation. (Thermal noise is not an important factor in ionization sensors.)

A microphone control, shown in the lower right corner of the rear plate, is unique because it is a "live microphone" attached to a separate impact plate having $1 / 15$ the effective area of the main microphone plate. Thus the control is exposed to the same environment as the main microphone, including impacts by cosmic dust; an approximate ratio of $1: 15$ would be expected between impacts on the control and impacts on the main microphone sensor.

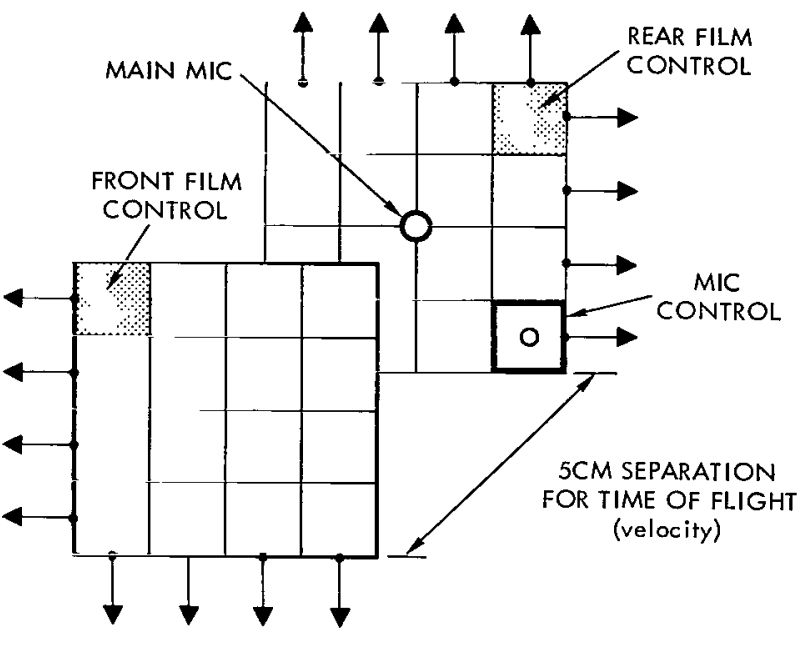

Figure 4-Cosmic dust sensor control locations.

As mentioned earlier, the acoustical sensors are designed to perform an in-flight study on the reliability of the microphone as a cosmic dust sensor and to serve as an impact sensor for this particular satellite.

\section{CALIBRATIONS}

Extensive calibrations have been performed on the sensors using a $2-\mathrm{MeV}$ electrostatic accelerator. Unfortunately the particles used for calibration have been limited to high-density, hard spheres of iron $\left(10^{-13} \mathrm{gm}<\right.$ mass $\left.<10^{-9} \mathrm{gm}\right)$ having velocities merely approaching the low end of the meteoroid velocity spectrum ( 2 to $10 \mathrm{~km} / \mathrm{sec}$ ). Accordingly, when considering the sensitivities of the sensors as derived from these calibrations, the potential discrepancies that may become manifest in subsequent measurements in space when the sensors are exposed to projectiles of diverse density, structure, composition, and velocity must be considered.

The plasma sensors respond nearly linearly to the particle's kinetic energy over the limited particle parameter range specified above for the laboratory simulator. The acoustical sensors respond to the particle's momentum for that same particle range.

The threshold sensitivity of the front film sensor array to laboratory particles is $0.6 \mathrm{erg}$. TOF is registered for laboratory particles having kinetic energies of $1.0 \mathrm{erg}$ or greater. The electronics of the TOF sensor are design-limited to particles having velocities ranging from 2 to $72 \mathrm{~km} / \mathrm{sec}$.

Hypervelocity particles passing through the " $\mathrm{A}$ " film are decelerated in inverse proportion to their kinetic energy (for a velocity range of 2 to $10 \mathrm{~km} / \mathrm{sec}$ ). For particles having the minimum energy required to exhibit time of flight $(1.0 \mathrm{erg})$, the deceleration is 40 percent; deceleration drops to 5 percent for particles having 10 ergs. 
In-flight calibration is provided and initiated by ground command. Two different formats of simulated data pulses are alternately presented to the input of each of the amplifier systems to check the condition of the electronics and the plasma sensors. The two formats alternately provide a high and a low amplitude pulse to monitor the lower and upper sensitivities of the amplifiers. The "A" and " $B$ " film pulses are appropriately spaced and in proper sequence to monitor the TOF electronics and solar aspect electronics. All accumulators advance with in-flight calibration.

In addition to the electronic monitors, the in-flight calibration provides a check on the physical condition of the plasma sensors. The positive pulse to the front and rear film amplifiers is also impressed upon the film. Because of capacitative crosstalk between the film and its corresponding grid, the high-amplitude calibration pulse is detected and amplified sufficiently in the grid amplifier to be displayed in readout. If the thin films have been torn and have become shorted to the grids, or have been partially or totally removed from their supports, the capacitative crosstalk will be altered and its effect will be displayed in the readout of calibration. Admittedly the same crosstalk will also appear for plasma current pulses resulting from impacts by high-energy particles, but only for those plasma pulses corresponding to the extreme energy range of the sensors.

\section{EXPERIMENT ELECTRONICS}

Figure 5 shows a block diagram of the experiment. A summer amplifier (or current driver amplifier) receives the positive-going pulse from each "A" film strip. After a gain of unity, the pulse divides into two separate paths. In one path it is amplified $(\mathrm{Vg}=15)$, its pulse height is analyzed, and its amplitude is recorded in the storage register. In the other path it is amplified $(\mathrm{Vg}=1000)$ and fed into a threshold one-shot. The output pulse performs three functions as shown:

(1) Its origin identification is impressed directly upon the storage register;

(2) It passes through the NOR gate and initiates the TOF measurement; and

(3) It is gated back to the threshold one-shot to inhibit any other "A" film pulse until the measurement has been completed.

An inhibit signal to the other three films is necessary to avoid capacitative crosstalk for highenergy impact signals. The "A" film pulse is pulse-height-analyzed and the results are stored in the register to await readout.

Positive-going pulses from the "B" film pass through a similar, but separate, electronic path with the following two exceptions: no PHA is performed on the " $\mathrm{B}$ " film pulse and the pulse is used to stop the TOF clock. If no " $B$ " film pulse follows an "A" film pulse, the TOF register goes to the full ( 63 counts) state and remains full until another event occurs.

Negative-going pulses from each of the " $\mathrm{A}$ " and " $\mathrm{B}$ " grids are amplified via separate units and ID-registered as shown. For simplicity, only one set of collector amplifiers is shown in the lower center area of Figure 5. Each film strip and grid strip in both the front and rear sensor arrays connects to its own separate amplifier system. 


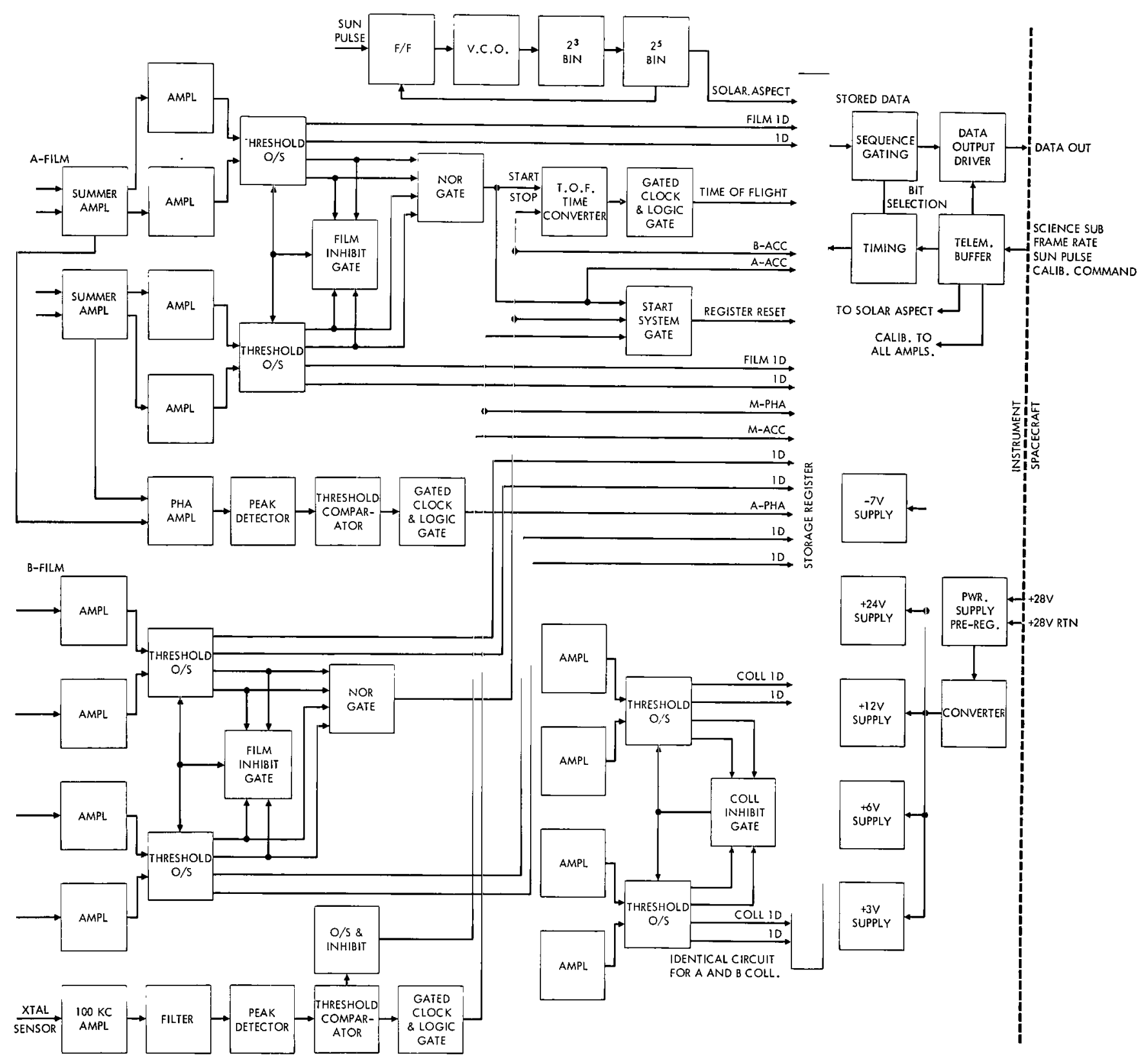

Figure 5-Block diagram of the experiment. 
The output signal from the crystal sensor (microphone), as it responds to impacts, is a ringing sinusoidal wave that increases to a maximum and then decays. After amplification in a tuned amplifier, the peak signal amplitude is used to advance the microphone accumulate, start the register reset (readout of register data), and record the amplitude of the impulse imparted to the microphone sensor plate. The one-shot and inhibit block shown in the microphone circuit inhibit further processing of subsequential microphone pulses until after the final pulse is placed in the storage register.

Pulses from the control microphone (not shown in the block diagram) follow a similar, but separate, electronic course with the exceptions that no PHA is performed and they do not trigger the register reset.

The solar aspect system starts counting from the time the sensor views the sun. The combined function of the flip-flop, the voltage-controlled oscillator, and the $2^{3}$ binary is to adjust the oscillator frequency continually to correlation with the spacecraft spin period so that the $2^{5}$ binary will read out 63 bits per revolution.

\section{TELEMETRY-BIT LAYOUT}

The data are displayed as 48 bits on four 6-bit words. This is accomplished by alternately displaying the data in the two formats shown in Figure 6 as the " $\mathrm{O}$ " frame and " 1 " frame.

The first bit in each frame identifies the frame. The next 8 bits in the " $O$ " frame identify the "A" film strip and "B" grid column affected by a cosmic dust particle impact. Bits 10 and 11 record the number of events measured by the control microphone. Six bits are assigned to TOF for projectiles in the velocity range 2 to $72 \mathrm{~km} / \mathrm{sec}$, which corresponds to a TOF range of $2.5 \times 10^{-5}$ to $7 \times 10^{-7} \mathrm{sec}$. Any "A" film event initiates the start of a $4-\mathrm{MHz}$ clock that is stopped by either a "B" film event or a filled register of 63 bits. A solar-aspect counter uses the next 6 bits of frame "O". This device starts its count upon each revolution of the spacecraft (1 rps) at a time when the sun sensor sees the sun. The last bit in frame "O" provides an experiment parity check.

The next 8 bits following frame ID in frame " 1 " are used for " $B$ " film strip and " $B$ " grid column identification for the rear sensor array. A single bit is used to indicate signal noise that may have occurred during PHA of any "A" film event or microphone event. Bits 11 and 12 of frame "1" register the total number of main-microphone events; bits 13 and 14 register the accumulated number of " $B$ " film events. The "A" film PHA and microphone PHA are registered on the next 6 bits. The remaining 4 bits are assigned to the display of accumulated "A" film hits. All of the data on both formats remain, and are repetitively displayed, until an event occurs involving the "A" film, the "B" film, or the microphones.

\section{SUMMARY}

The Pioneer 8 cosmic dust experiment evolved from two decades of in situ measurements aimed at defining the cosmic dust environment in the solar system. Its design represents the 


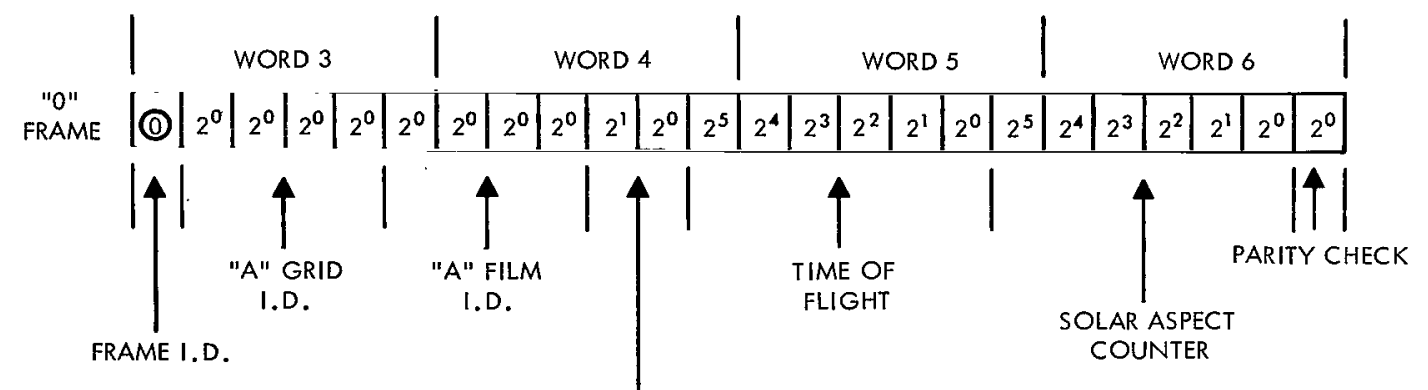

ANY MICROPHONE NOISE

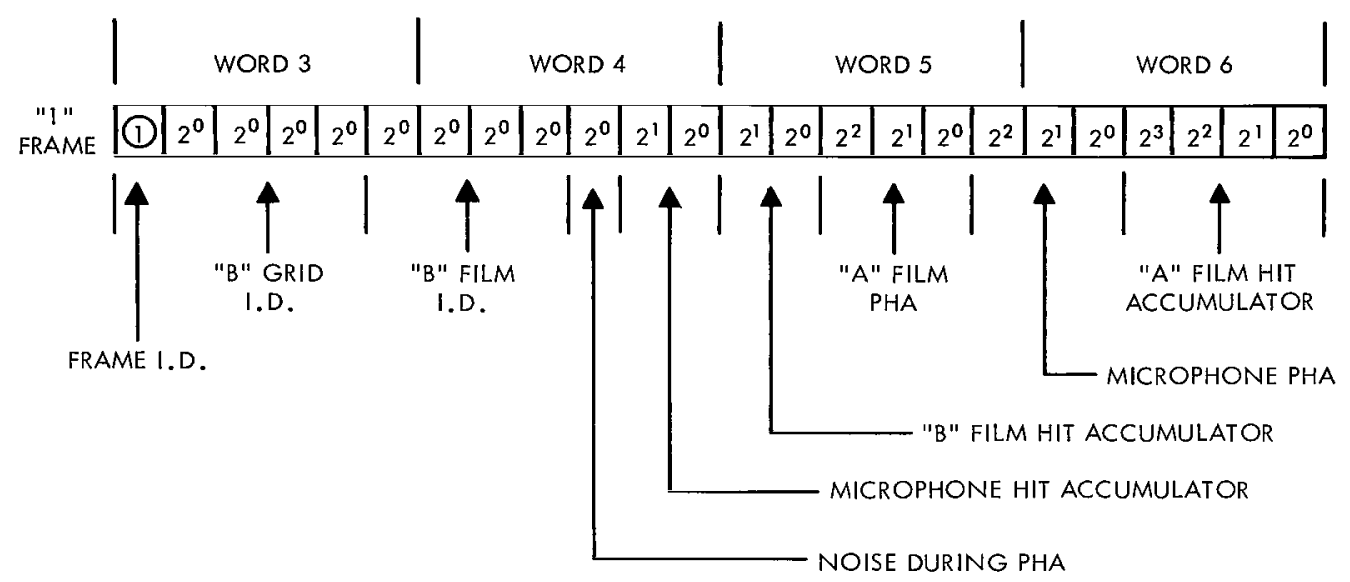

Figure 6-Telemetry bit assignment.

recognition of several existing problems which are peculiar to these measurements:

1. A lack of definitive data concerning the structure, composition, mass density, etc. of the spatial particle

2. The presently limited range of physical parameters available in laboratory-simulated cosmic dust particles

3. The response of acoustical sensors to the real cosmic dust particle in the environment of that particle.

It is anticipated that the combination of coincidence sensors in the experiment, the unique controls and monitors in the experiment, and the long operational lifetime of Pioneer 8 will provide data that will contribute significantly to our understanding of the meteoroid environment.

Goddard Space Flight Center

National Aeronautics and Space Administration

Greenbelt, Maryland, September 27, 1968

811-12-02-01-51 
National Aeronautics and Space Administration

Washington, D. C. 20546

OFFICIAL BUSINESS
FIRST CLASS MAIL

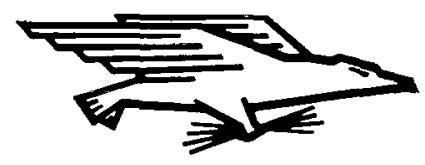

POSTAGE AND FEES PAID NATIONAL AERONAUTICS AND SPACE ADMINISTRATION

$$
\begin{aligned}
& 12000155 \quad 513115 \quad 0917800903
\end{aligned}
$$

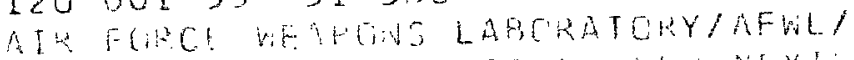

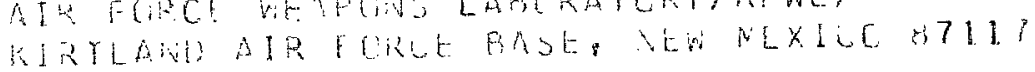

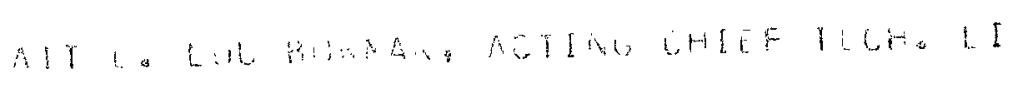

POSTMASTER:

"The aeronatical and space activities of the United States shall be conducted so as to contribute. . . to the expansion of buman knowledge of phenomena in the atmosphere and space. The Administration sball provide for the widest practicable and appropriate dissemination of information concerning its activities and the results thereof."

- National Aeronautics and Space Act of 1958

\section{NASA SCIENTIFIC AND TECHNICAL PUBLICATIONS}

TECHNICAL REPORTS: Scientific and technical information considered important, complete, and a lasting contribution to existing knowledge.

TECHNICAL NOTES: Information less broad in scope but nevertheless of importance as a contribution to existing knowledge.

TECHNICAL MEMORANDUMS:

Information receiving limited distribution because of preliminary data, security classification, or other reasons.

CONTRACTOR REPORTS: Scientific and technical information generated under a NASA contract or grant and considered an important contribution to existing knowledge.
TECHNICAL TRANSLATIONS: Information published in a foreign language considered to merit NASA distribution in English.

SPECIAL PUBLICATIONS: Information derived from or of value to NASA activities. Publications include conference proceedings, monographs, data compilations, handbooks, sourcebooks, and special bibliographies.

\section{TECHNOLOGY UTILIZATION}

PUBLICATIONS: Information on technology used by NASA that may be of particular interest in commercial and other non-aerospace applications. Publications include Tech Briefs, Technology Utilization Reports and Notes, and Technology Surveys.

Details on the availability of these publications may be obtained from:

SCIENTIFIC AND TECHNICAL INFORMATION DIVISION

NATIONAL AERONAUTICS AND SPACE ADMINISTRATION

Washington, D.C. 20546 